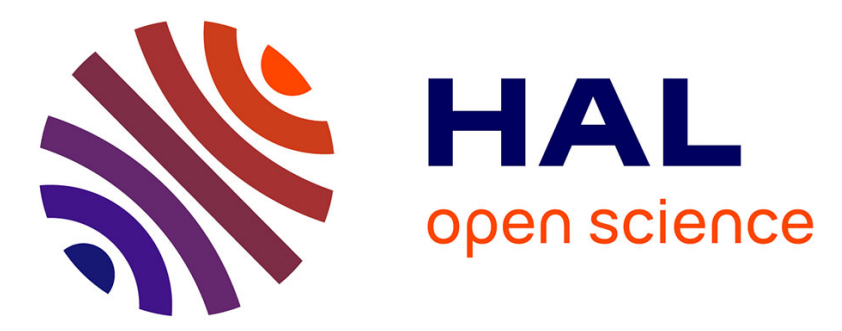

\title{
Topological edge modes with PT symmetry in a quasiperiodic structure
}

Nicolas X A Rivolta, Henri Benisty, Bjorn Maes

\section{To cite this version:}

Nicolas X A Rivolta, Henri Benisty, Bjorn Maes. Topological edge modes with PT symmetry in a quasiperiodic structure. Physical Review A : Atomic, molecular, and optical physics [1990-2015], 2017, 96 (2), pp.23864 - 23864. 10.1103/PhysRevA.96.023864 . hal-01611097

\section{HAL Id: hal-01611097 \\ https://hal-iogs.archives-ouvertes.fr/hal-01611097}

Submitted on 10 Oct 2017

HAL is a multi-disciplinary open access archive for the deposit and dissemination of scientific research documents, whether they are published or not. The documents may come from teaching and research institutions in France or abroad, or from public or private research centers.
L'archive ouverte pluridisciplinaire HAL, est destinée au dépôt et à la diffusion de documents scientifiques de niveau recherche, publiés ou non, émanant des établissements d'enseignement et de recherche français ou étrangers, des laboratoires publics ou privés. 


\title{
Topological edge modes with $\mathcal{P} \mathcal{T}$ symmetry in a quasiperiodic structure
}

\author{
Nicolas X. A. Rivolta, ${ }^{1, *}$ Henri Benisty, ${ }^{2}$ and Bjorn Maes ${ }^{1}$ \\ ${ }^{1}$ Micro- and Nanophotonic Materials Group, Faculty of Science, University of Mons, 20 Place du Parc, 7000 Mons, Belgium \\ ${ }^{2}$ Laboratoire Charles Fabry, Institut d'Optique, CNRS, Universite Paris-Sud, 2 Avenue Augustin Fresnel, 91127 Palaiseau, France
}

(Received 13 June 2017; published 30 August 2017)

\begin{abstract}
We investigate topological features of a one-dimensional photonic quasicrystal within the context of $\mathcal{P} \mathcal{T}$ symmetry. Via the scattering characteristics, we analyze various properties of a particular mirrored structure, which supports topological edge modes in its band gaps. These interface modes display a nontrivial dependence on the quasiperiodic geometry, even in a passive system. Subsequently, the tailored addition of gain and loss generates curious $\mathcal{P} \mathcal{T}$-like features. For example, the quasicrystal high density of modes leads to complicated mode-merging behaviors between edge and band modes, such as the symmetry recovery phenomenon. Furthermore, anisotropic transmission resonances (connected with unidirectional invisibility) are also present, but they display richer patterns in comparison to previously studied periodic structures. Additionally, we examine lasing effects in detail, with numerics and a simple Fabry-Pérot model. The large variety of mode-merging behaviors opens the way to laser resonance engineering.
\end{abstract}

DOI: 10.1103/PhysRevA.96.023864

\section{INTRODUCTION}

Parity-time $(\mathcal{P} \mathcal{T})$ symmetry is a blossoming field that brings opportunities to photonics by tailoring gain and loss [1-3]. It originates from quantum mechanics and exploits non-Hermitian Hamiltonians, which under some conditions may possess purely real spectra [4,5]. Implemented in optics, $\mathcal{P} \mathcal{T}$ symmetry leads to structures with a balance between gain and loss. This balance typically means that the refractive index satisfies a symmetry relation $n(x)=n^{*}(-x)$. Therefore, the loss is exploited as a crucial element and is not an unwanted feature anymore [6]. One of the most interesting characteristics is $\mathcal{P} \mathcal{T}$-symmetry breaking, a phase transition where eigenvalues flip from purely real to complex-conjugate pairs at what is called an exceptional point [7], marking a clear boundary between various optical behaviors.

Parity-time symmetry has been successfully harnessed in many photonic structures, such as directional couplers $[8,9]$, switching devices [10,11], plasmonic structures [12], Bragg reflectors [13], microring resonators and microdisks [14,15], gratings [16-18] (or even phononic grating [19]), quasicrystals [20-22], robust transport [23,24], and others [25,26]. The field of topology, on the other hand, examines the conservation of mathematical or physical properties under continuous deformations. It expanded towards the development and comprehension of topological insulators [27] and it opens opportunities in many fields and in particular in photonics [28]. One of the intriguing promises is a unidirectional waveguide that allows light to travel through an imperfect structure without suffering from backreflection. Topological effects are available in many photonic systems, for example, in photonic crystals, coupled resonators, metamaterials, and quasicrystals [29]. In particular, one-dimensional (1D) quasiperiodic structures [30-32] with or without a defect [33] have a proven interest as a topological playground [34].

Parity-time symmetry has been employed to obtain a topological state [35-37] or to tailor such states $[38,39]$ within a

\footnotetext{
*nicolas.rivolta@umons.ac.be
}

1D structure. Here we associate $\mathcal{P} \mathcal{T}$ symmetry and topology in a 1D quasicrystal device to observe their intriguing combined characteristics. With the capabilities of $\mathcal{P} \mathcal{T}$ symmetry to act on band gaps, we examine how gain and loss affect edge modes that are lying inside the gaps. With the extended gain-loss features, possibilities are suggested for engineering the lasing spectrum (gain-frequency relation at threshold).

We consider as a model system a quasicrystal consisting of two abutted Fibonacci sequences presenting topological edge modes, recently introduced for the passive regime [40,41] and within a polaritonic framework (even though [40] hinges on Fabry-Pérot models, we work here entirely with the more popular ordinary wave Fresnel reflection and we briefly discuss the differences for the passive system). We present the curious mode-merging behaviors displayed by the different types of modes in this quasicrystal when $\mathcal{P} \mathcal{T}$ symmetry is added. Among other typical $\mathcal{P} \mathcal{T}$ phenomena, for example, the symmetry-recovery effect [42] appears. In addition, as in other 1D $\mathcal{P} \mathcal{T}$ structures, we detect anisotropic transmission resonances [43], or single-sided reflections, but in a much more complex pattern than previously noticed in periodic structures. Furthermore, due to the presence of gain, we get a family of lasing resonances, which we are able to describe with a simple Fabry-Pérot model.

We portray the passive structure in Sec. II, with a calculation of its transmittance and an interpretation of its intrinsic topological properties. Subsequently, we add gain and loss in Sec. III, in order to investigate the global (Sec. III A) or local (Sec. III B) interplay between $\mathcal{P} \mathcal{T}$ - and topologyrelated characteristics, before discussing the lasing spectra in Sec. III C and summarizing in Sec. IV.

\section{TOPOLOGICAL FIBONACCI SEQUENCE}

We study the scattering properties of an open 1D photonic quasicrystal structure composed of two parts stitched together. In this section we examine only the passive properties (no gain or loss). The two parts are Fibonacci sequences, which are quasiperiodic and composed of an alternation of two letters $A$ and $B$, representing two different materials [Fig. 1(a)]. 
(a)
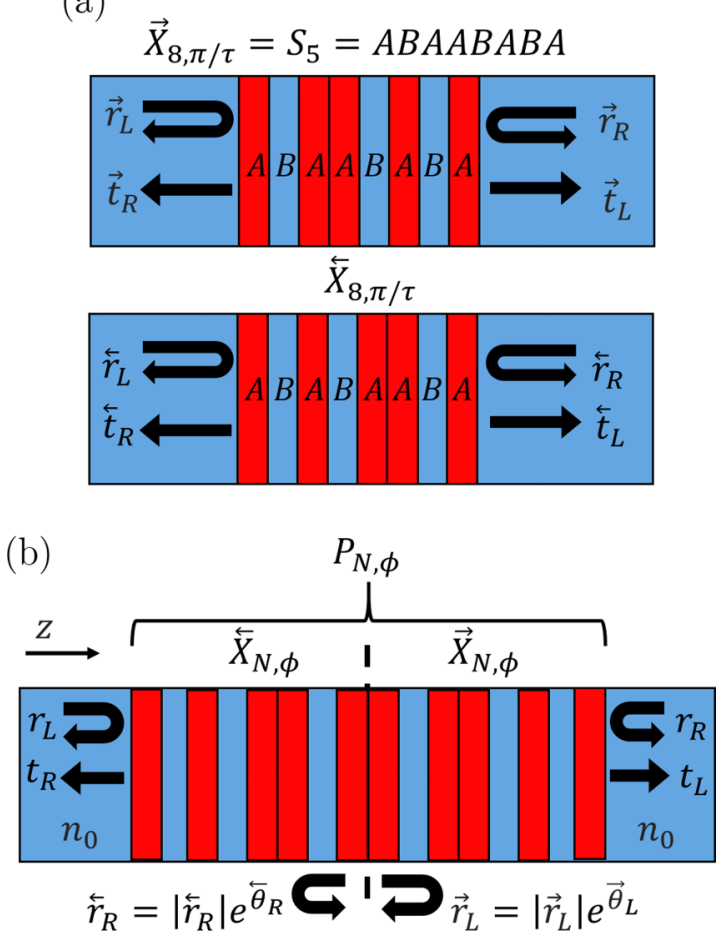

FIG. 1. (a) Geometry of the Fibonacci sequences $\overleftarrow{X}_{N, \phi}$ and $\vec{X}_{N, \phi}$ with their implementations into a photonic quasicrystal. (b) Geometry of the structure $P_{N, \phi}$ with the convention for the right and left transmission and reflection.

The traditional mechanism to generate a Fibonacci sequence is by using an inflation and substitution algorithm $\sigma$. Here $\sigma$ applies to $A$ and $B$ according to $\sigma(A)=A B$ and $\sigma(B)=A$ and its application to more than one letter obeys the relation $\sigma(Y Z)=\sigma(Y) \sigma(Z)$ [e.g., $\sigma(A B)=\sigma(A) \sigma(B)=$ $A B A]$. Consecutive applications of $\sigma$ give rise to a Fibonacci sequence $S_{j}=\sigma^{j}(B)$ (with $j \in \mathbb{N}$ ), whose length is the Fibonacci number $F_{j>1}=F_{j-1}+F_{j-2}\left(\right.$ with $\left.F_{0}=F_{1}=1\right)$. When $j \rightarrow \infty$ the ratio $F_{j+1} / F_{j} \rightarrow \tau$, with $\tau=(1+\sqrt{5}) / 2$ the golden ratio [44]. The infinite chain $S_{\infty} \equiv \lim _{j \rightarrow \infty} S_{j}$ is quasiperiodic and self-similar under the substitution process [45]. This sequence can be roughly compared to a periodic succession of $A$ 's and $B$ 's with pseudorandomly added $A$ letters [see $S_{5}$ in Fig. 1(a)].

A convenient way to describe $S_{j}$ is to use the two-valued function $\chi_{n, \phi}$, whose values \pm 1 are identified as $A(+1)$ and $B(-1)$ :

$$
\chi_{n, \phi}=\operatorname{sgn}[\cos (2 \pi n / \tau+\phi)-\cos (\pi / \tau)],
$$

with $n \in \mathbb{N}_{0}$ and a degree of freedom $\phi$ that is $2 \pi$ periodic. In this paper we will use this second construction method to define a more general parametrized structure. This angular degree of freedom $\phi$ is irrelevant for the infinite chain, but relevant for a finite segment $\vec{X}_{N, \phi}=\left[\chi_{1, \phi} \chi_{2, \phi} \cdots \chi_{N, \phi}\right]$ of $\vec{X}_{\infty, \phi}$. One can show that the finite segment $S_{j}=\sigma^{j}(B)$ is the same as $\vec{X}_{N, \phi}$, if $\phi=\pi / \tau$ and $N=F_{j}$. However, in general in this paper $\phi \neq \pi / \tau$ and the two constructions are

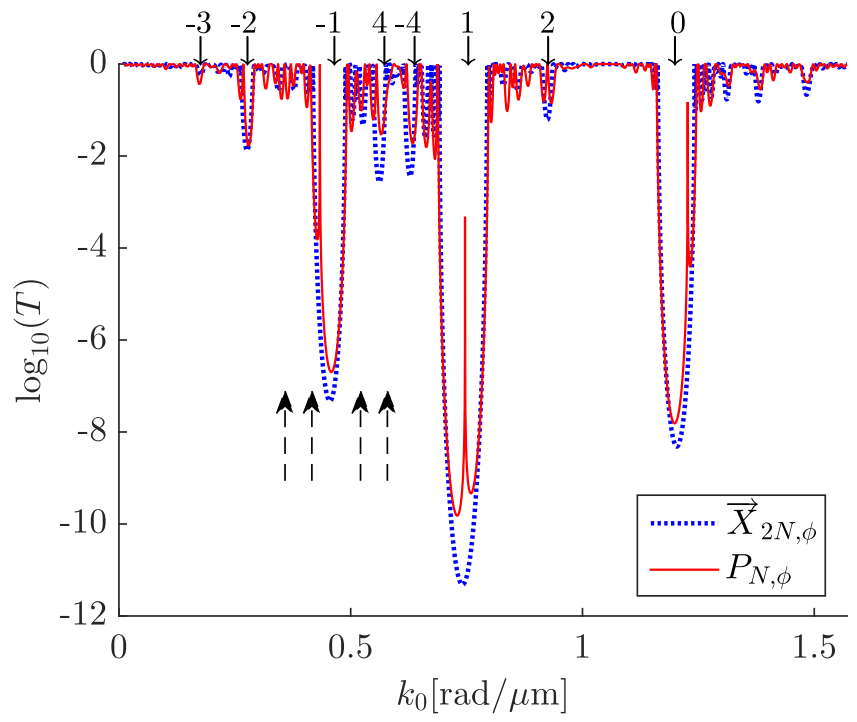

FIG. 2. Transmission of $\vec{X}_{2 N, \phi}$ (dotted blue) and $P_{N, \phi}$ (solid red) with $N=50$ and $\phi=3 \mathrm{rad}$. The black arrows on top denote the positions of the band gaps given by Eq. (2) with the corresponding $m$ values. The four dashed black arrows below indicate the $k_{0}$ values of the profiles in Fig. 3.

not equivalent. The irrationality of $\tau$ ensures that $\vec{X}_{N, \phi}$ is quasiperiodic [20].

Finally, there is also a geometrical construction of $\vec{X}_{N, \phi}$ possible via the cut-and-project method. This visualization principle links to one of the computational methods for calculating the spectrum and eigenstates of a quasicrystal, by solving Maxwell's equations in higher dimensions (i.e., the superspace method) [44].

Our structure $P_{N, \phi}$, which we call a twinned inverted Fibonacci sequence (TIFS), is composed of two symmetric abutted segments, the reversed $\overleftarrow{X}_{N, \phi}=\left[\chi_{N, \phi} \chi_{N-1, \phi} \cdots \chi_{1, \phi}\right]$ and $\vec{X}_{N, \phi}$ [see Fig. 1(b)], with the aim to disrupt the spectral properties of the Fibonacci sequence and thus to induce defect modes. For photonic implementation, $A$ and $B$ (or \pm 1 ) correspond to two different material layers with the same length $L=1 \mu \mathrm{m}$ and refractive indices $n_{A}=3$ and $n_{B}=2$, respectively. The outside refractive index is $n_{0}=2$.

We investigate the properties of the TIFS structure $P_{N, \phi}$ through numerical simulations (with CAMFR [46]) of the transmission $T=\left|t_{L}^{2}\right|=\left|t_{R}^{2}\right|$, with $t_{L}$ and $t_{R}$ the left and right transmission amplitudes, respectively, and the left and right reflection $R_{L, R}=\left|r_{L, R}^{2}\right|$, with $r_{L, R}$ the left and right reflection amplitudes [see Fig. 1(b) for conventions]. For $\overleftarrow{X}_{N, \phi}$ and $\vec{X}_{N, \phi}$ we use the same conventions for transmission or reflection with an extra left or right arrow [e.g., $\vec{r}_{L}$ for the left reflection amplitude of $\vec{X}_{N, \phi}$; see Fig. 1(a)]. The transmission is the same regardless of the input side because of reciprocity.

Figure 2 shows the transmission of $P_{N, \phi}$ and the related nonmirrored segment $\vec{X}_{2 N, \phi}$ (2N in order to have the same number of layers as $P_{N, \phi}$ ) as a function of $k_{0}=2 \pi / \lambda_{0}$, with $\lambda_{0}$ the vacuum wavelength of an incident perpendicular plane wave. Similar to a periodic crystal, quasiperiodicity opens a 


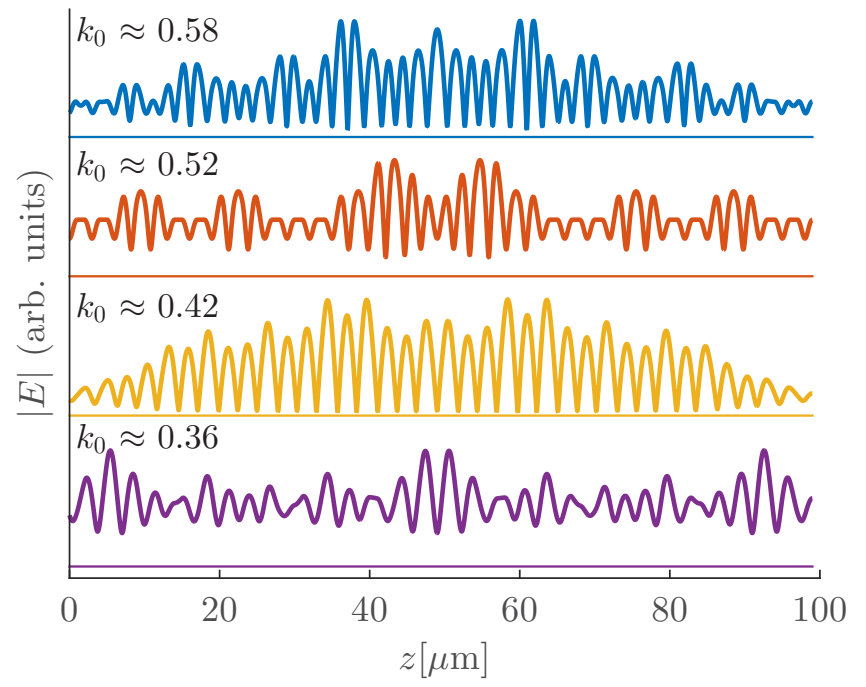

FIG. 3. Field profiles of the band modes of the TIFS structure $P_{N, \phi}$ as a function of the position $z$ with $N=50$ and $\phi=5 \mathrm{rad}$. The modes at $k_{0} \approx 0.52$ and $0.36 \mathrm{rad} / \mu \mathrm{m}$ are located in the middle of a band. The modes at $k_{0} \approx 0.58$ and $0.42 \mathrm{rad} / \mu \mathrm{m}$ are located at the edge of a band. These $k_{0}$ are indicated by dashed black arrows in Fig. 2.

series of band gaps for both structures in the same ranges of $k_{0}$.

We can locate the theoretical positions of these band gaps via the gap-labeling theorem $[29,45,47,48]$

$$
k_{\text {gap }}=[n+\bmod (m / \tau, 1)]\left(2 \pi / D n_{\mathrm{av}}\right),
$$

with $m \in \mathbb{Z}, n \in \mathbb{N}, D=2 L$ the basic pair thickness of our system, and $n_{\mathrm{av}}$ the average refractive index. We mark the band gaps using this equation with black arrows and the corresponding $m$ number on top in Fig. 2.

If we compare these quasiperiodic spectra to the band diagram of a periodic succession of $A$ and $B$ (not shown), the gap around $k_{0} \approx 1.2 \mathrm{rad} / \mu \mathrm{m}(m=0)$ appears in both periodic and quasiperiodic structures: It corresponds to the natural first band gap (with $n=1$ ) of the periodic structure and to the smallest physical distance for a constructive round-trip. All the other gaps of the quasiperiodic structure thus open in a passband of the periodic structure (e.g., the first pass band $n=0$ of the periodic structure is for $k_{0}<1.2 \mathrm{rad} / \mu \mathrm{m}$ ).

It is interesting to note that an infinite quasiperiodic sequence opens an infinite number of band gaps and the transmission spectrum becomes fractal $[45,48]$. Here for a finite sequence, we observe only the larger band gaps with smaller $|m|$.

Each transmission peak inside and outside the band gaps indicates a mode of the structure. Turning to the TIFS $P_{N, \phi}$ (solid red curve in Fig. 2), we observe some additional peaks of transmission inside the band gaps in comparison to the transmission of $\vec{X}_{2 N, \phi}$ (dotted blue curve). As $P_{N, \phi}$ is not an exact Fibonacci sequence but is the result of stitching two sequences, this configuration creates an interface in the structure at the mirror plane. This interface allows for one or more additional modes to appear in the band gaps.

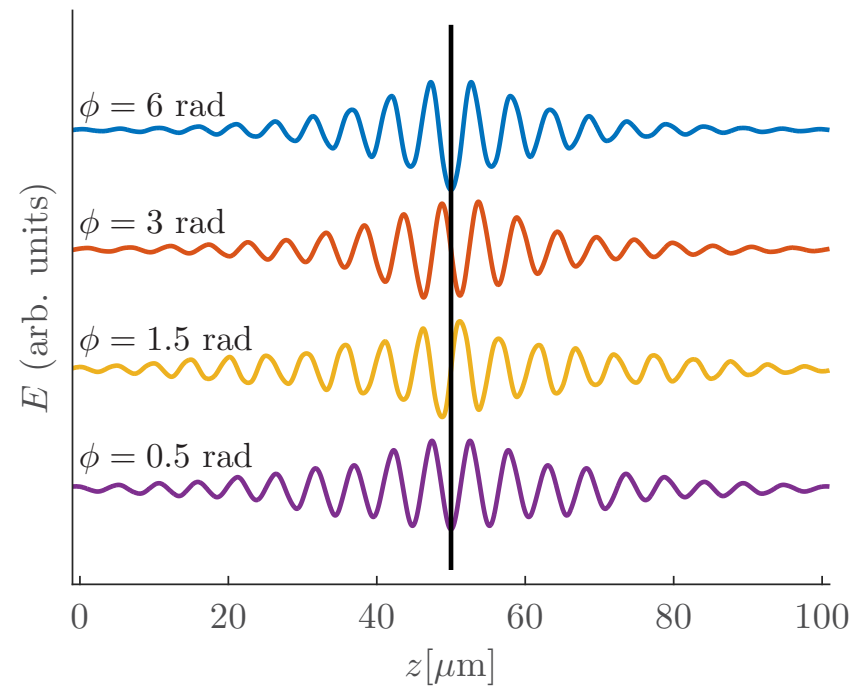

FIG. 4. Electrical $(E)$ field profiles of the interface mode as a function of the position $z$ [see Fig. 1(b)] for varying $\phi$. The structure is between $z=0$ and $100 \mu \mathrm{m}$; the symmetry plane is indicated by the black line. The interface mode is located in the band gap around $k_{0} \approx 0.45 \mathrm{rad} / \mu \mathrm{m}$ and $m=-1$ in Fig. 2 .

We show in Fig. 3 the profiles of four band modes of $P_{N, \phi}$ around the band gap with $m=-1$. They display different shapes but are all symmetric with respect to the central mirror plane of $P_{N, \phi}($ at $z=50 \mu \mathrm{m})$. The two modes with $k_{0} \approx 0.58$ and $0.42 \mathrm{rad} / \mu \mathrm{m}$ possess fewer modulations nodes than the others and thus can be considered as more fundamental (more details in Sec. III A). All these profiles are also spatially extended over the whole structure.

The latter is in contrast with the field profiles of the TIFS interface modes; we show these modes for the gap with $m=-1$ in Fig. 4 for different values of $\phi$. An interface mode is confined to the central mirror plane for every value of $\phi$ and this property is general for all band-gap modes. The interface mode profiles also present a symmetry (e.g., for $\phi=0.5$ and 6 rad in Fig. 4) or an antisymmetry $(\phi=1.5$ and $3 \mathrm{rad})$; more details will be provided below. Finally, larger band gaps lead to more confined interface modes (not shown) with narrower bandwidths.

We focus on the transmission around two of the lowerfrequency band gaps $\left[k_{0} \approx 0.45 \mathrm{rad} / \mu \mathrm{m}\right.$ and $m=-1$ in Fig. 5(a) and $k_{0} \approx 1.2 \mathrm{rad} / \mu \mathrm{m}$ and $m=0$ in Fig. 5(b)], varying $\phi$ and $k_{0}$. We do not examine the larger band gap with $m=1$, as it encounters the same topological properties as for $m=-1$. Moreover, in a larger band gap the interface mode is narrower, so a finer grid of $k_{0}$ (higher computational cost) is needed. The interface mode in the band gaps describes one [Fig. 5(b)] or two [Fig. 5(a)] cycles as a function of $\phi$, respectively. Along these cycles the interface mode performs some spectral jumps for definite values of $\phi$. These jumps are the consequence of a letter switch in the sequence $\vec{X}_{N, \phi}$ (and symmetrically in $\overleftarrow{X}_{N, \phi}$ ), when $\phi$ varies [see Fig. 5(c)]

More in detail, each letter of $\vec{X}_{N, \phi}$ flips from $B$ to $A$ [red crosses in Fig. 5(c)] at a different value of $\phi$ [see Eq. (1)]. In addition, at this value of $\phi$, the next letter in the sequence (except for the first one) also does a reverse flip from $A$ to $B$ [blue circles in Fig. 5(c)]. As $\overleftarrow{X}_{N, \phi}$ mirrors $\vec{X}_{N, \phi}$, these 

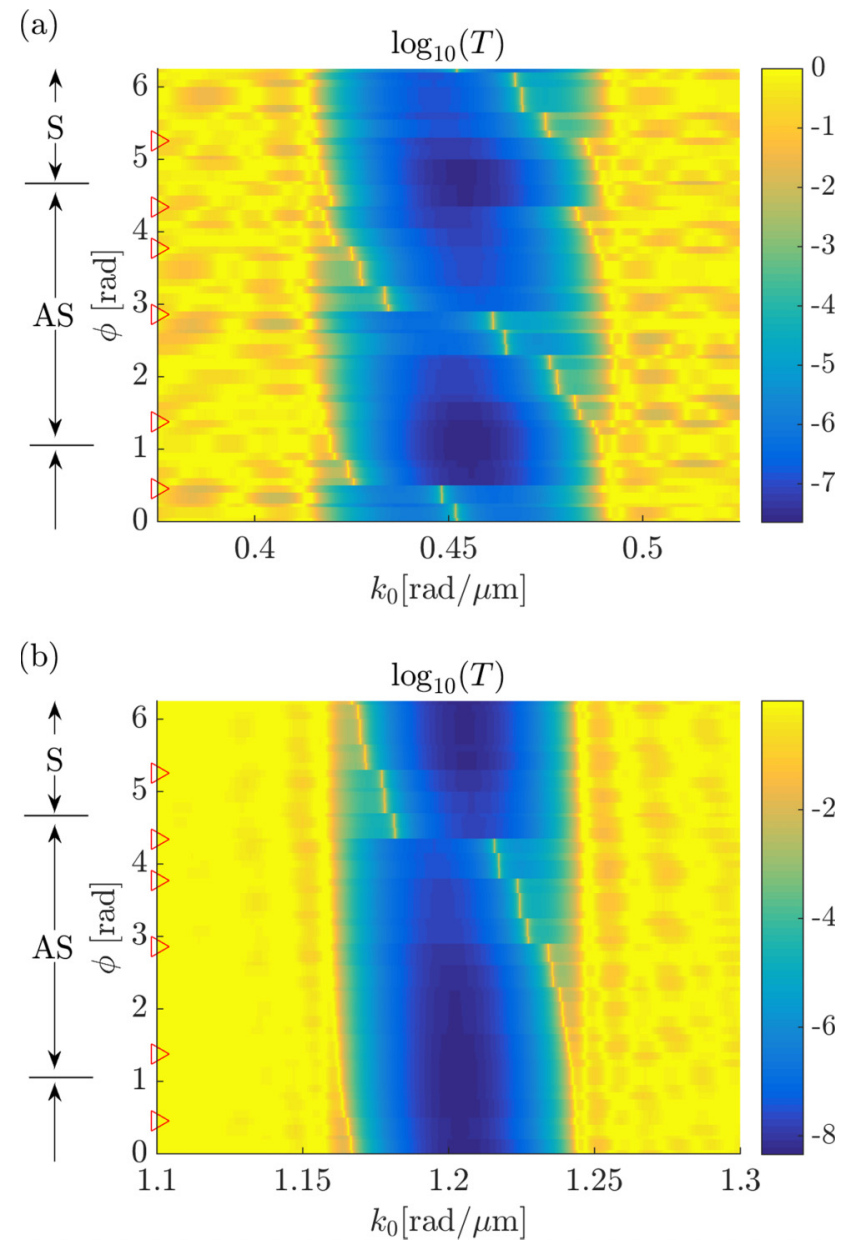

(c)

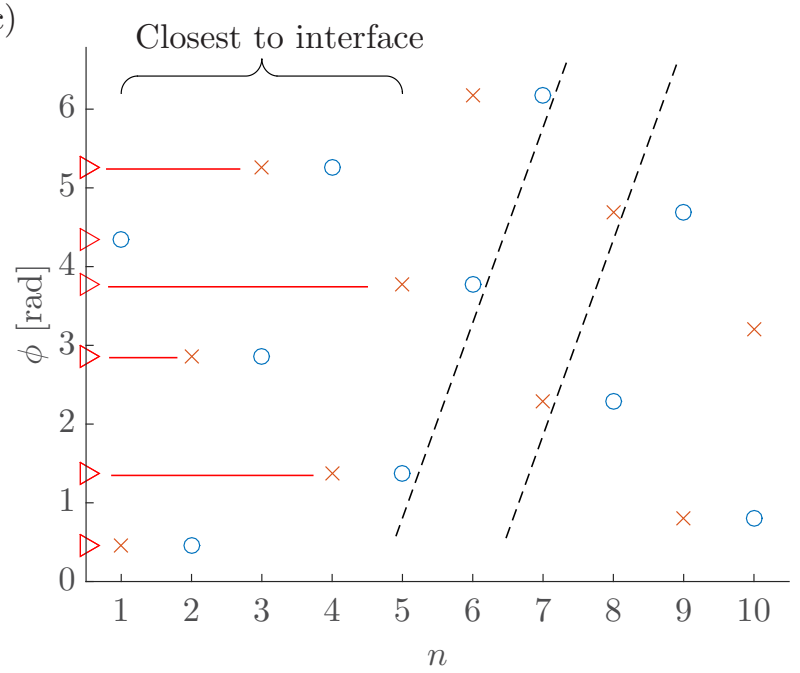

FIG. 5. (a) and (b) Transmission ( $\log _{10}$ scale) of the TIFS structure $P_{N, \phi}$ as a function of $k_{0}$ and $\phi$ with $N=50$. The red triangles on the left axis indicate the values of $\phi$ where at least one of the first (last) letters of $\vec{X}_{N, \phi}\left(\overleftarrow{X}_{N, \phi}\right)$ flips, leading to a spectral (horizontal) jump of the interface mode. The AS and $\mathrm{S}$ zones stand for the $\phi$ regions where the interface mode profiles are antisymmetric or symmetric, respectively. (c) The $\phi$ values where a letter flips as a function of the letter position $n$ in the sequence $\vec{X}_{N, \phi}$ (or symmetrically at the position $N-n+1$ in $\overleftarrow{X}_{N, \phi}$ ). The red crosses show the flips from $B$ to $A$ and vice versa for the blue circles. conclusions are also valid for the inverse sequence. As the interface mode is confined to the center of the TIFS structure $P_{N, \phi}$, the flipping of the first letters of $\vec{X}_{N, \phi}$ (or the last ones of $\overleftarrow{X}_{N, \phi}$ ) induce a larger jump than those at the extremities of the chain $P_{N, \phi}$ (with negligible field amplitude). We indicate with red triangles in Fig. 5 the $\phi$ values where one of the five first (last) letters of $\vec{X}_{N, \phi}\left(\overleftarrow{X}_{N, \phi}\right)$ flips, indeed leading to a significant spectral jump of the interface mode.

Furthermore, we indicate that the red crosses and blue circles are situated along lines [black dashed lines in Fig. 5(c)]. In addition, the $\phi$ separation [vertical distance in Fig. 5(c)] between the same kind of flip ( $A$ to $B$ or $B$ to $A$ ) of two successive letters is always equal to $2.4 \mathrm{rad}$ [so the vertical distance between two red crosses or between two blue circles in Fig. 5(c)]. The incommensurability between this flip period and the $\phi$ period prevents these flips from ever occurring two times at the exact same value of $\phi$.

The number of cycles of the interface mode eigenvalues for a $2 \pi$ path of $\phi$ is determined by the winding number $w\left(k_{0}\right)$ of the band gap:

$$
w\left(k_{0}\right)=\frac{1}{2 \pi} \int_{0}^{2 \pi} \frac{\partial \theta_{\mathrm{cav}}\left(k_{0}, \phi\right)}{\partial \phi} d \phi,
$$

with $\theta_{\text {cav }}=\vec{\theta}_{L}+\overleftarrow{\theta}_{R}$ the sum of the phases of the reflection coefficients at the inner boundaries of $\vec{X}_{N, \phi}$ and $\overleftarrow{X}_{N, \phi}$ [see Fig. 1(b)]. The winding number has to be evaluated at a particular $k_{0}$, but one obtains the same value for any $k_{0}$ in the band gap. Note that without gain and loss $\vec{\theta}_{L}=\overleftarrow{\theta}_{R}$. The winding number represents the number of times a closed curve [defined by $\theta_{\text {cav }}\left(k_{0}, \phi\right)$ here] travels around a point. This number is also connected with the gap Chern number [49], the characteristic number that links band structure and topology [50].

If we check the interface mode field profile (Fig. 4), we observe that it keeps an antisymmetric pattern along a cycle in the band gap [AS region between $\phi \approx 1.4$ and $4.3 \mathrm{rad}$ in Fig. 5(a)]. For the second cycle $[S$ region between $\phi \approx 4.3$ and $1.4+2 \pi$ rad in Fig. 5(a)] the pattern is symmetric. The profile symmetry of the edge mode flips from symmetric to antisymmetric (or vice versa) when the mode begins another cycle or spectral crossing through the band gap. If the number of cycles is odd but at least three, the profile symmetry still flips between consecutive cycles. However, the single-cycle mode that crosses $\phi \approx 4.3$ [as in Fig. 5(b)] flips the symmetry in the middle rather than at the start or the end regions of a cycle. For example in Fig. 5(b), the edge mode is antisymmetric in the AS region below $\phi \approx 4.3 \mathrm{rad}$ and symmetric in the $\mathrm{S}$ region beyond this $\phi$ value while it is still in the same cycle. This value of $\phi$ corresponds to a change of the very first letter of $\vec{X}_{N, \phi}$ from $A$ to $B$ [Fig. 5(c)], i.e., a strong change at the core of the resonant cavity-like interface region leading to the possibility of a symmetry switch.

Compared to [40], we follow a fairly similar path as regards all generic properties of the TIFS structure (the phase, the interface mode, etc.). However, our approach has three distinctive features.

First, we deal with simple interfaces of known Fresnel reflection. This is welcome to expand the work to the optics domain. The Supplemental Material of [40] makes good use 


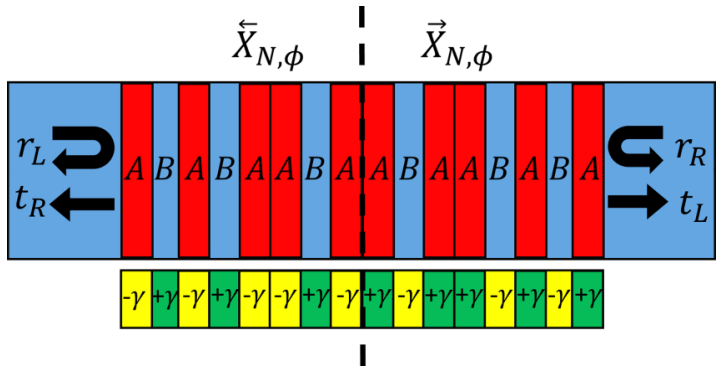

FIG. 6. Sketch of the TIFS structure $P_{N, \phi}$ with the gain and loss factor $\gamma$. All $A$ 's on left have gain $(-\gamma)$, all $B$ 's on the left have loss $(+\gamma)$, and vice versa for the right side.

of a Fabry-Pérot model, but does not give a self-contained view of the conditions for the local reflection factor. The actual reflection factor of a polaritonic Bloch wave in a stub-modulated waveguide like that of [40] is not an intuitive quantity, even if it boils down to a classical wave problem. So the size of the gap may be more directly related to optical realizations based on our model.

Second, we give a more explicit account of the role of the phase $\phi$ and the letter change in Fig. 5(c).

Third, we deal with an explicit transmission experiment and not a system bounded by hard walls. We hypothesize that this relaxes the phase condition(s) as the mode does not always decay quickly enough to be fully decoupled from the boundary, e.g., when the mode is close to a band-gap edge. This is our best clue as regards the reason why we observe a special case where the symmetry changes in the middle [Fig. 5(b)], thus contradicting Eq. (3) of [40]. An open optical system with well-mastered Fresnel reflection is plausibly a good basis to explore transmission and all the modes of these new structures.

\section{III. $\mathcal{P} T$ SYMMETRY}

Now we introduce gain and loss in a balanced fashion on both sides of the mirror plane. Therefore, in $\overleftarrow{X}_{N, \phi}$ all letters $A$ experience gain $\left(n_{A}=3-j \gamma\right)$ and all letters $B$ experience loss $\left(n_{B}=2+j \gamma\right)$, with $\gamma$ the gain-loss factor. For $\vec{X}_{N, \phi}$ we introduce the complex conjugates $\left(n_{A}=3+j \gamma\right.$ and $n_{B}=$ $2-j \gamma$; see Fig. 6). In this way the whole TIFS structure $P_{N, \phi}$ is $\mathcal{P} \mathcal{T}$ symmetric, but each part individually is not. As there are more $A$ 's than $B$ 's, $\overleftarrow{X}_{N, \phi}$ presents more gain than loss (depending on the sign of $\gamma$ ) and vice versa for $\vec{X}_{N, \phi}$.

Note that the relatively large $\gamma$ values $(|\gamma| \in[0,0.5])$ that we explore are connected to the large index contrast $n_{B}-n_{A}$ and the limited $N=50$ of our simulations. More realistic $|\gamma|<0.005$ could result from a combination of weaker index contrast $\left|n_{B}-n_{A}\right| \sim 0.1$ and larger $N>50$.

\section{A. Global properties}

An interesting global transmission pattern arises as the gainloss value increases. We show $T$ of $P_{N, \phi}$ as a function of $k_{0}$ and $\phi$ for various $\gamma$ in Fig. 7. As we increase $\gamma$ from Fig. 7(a) to Fig. 7(d), saturated transmission peaks [in yellow (light gray)] appear. However, for even larger $\gamma$ they subsequently tend to disappear. This pattern indicates the typical merging
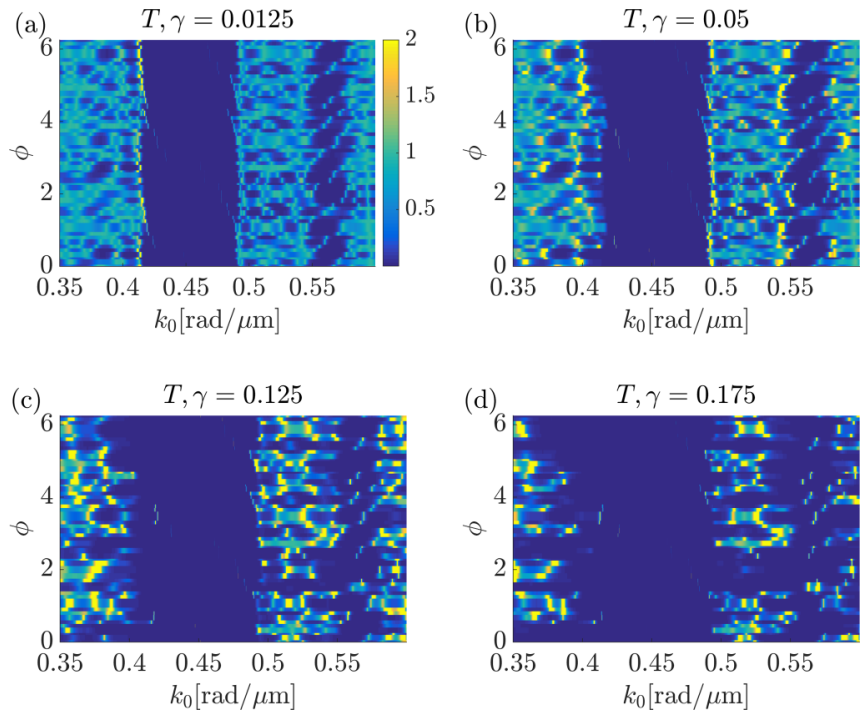

FIG. 7. Transmission $T$ (saturated to 2 for clarity) of $P_{N, \phi}$ as a function of $k_{0}$ and $\phi$ with $N=50$ and varying $\gamma$. The interface modes are less visible than in Figs. 5(a) and 5(b) due to the linear scale used here.

of two modes at an exceptional point under the action of $\mathcal{P} \mathcal{T}$ symmetry. Beyond the exceptional points, the transmission peaks disappear as the modes become complex (leading to dark blue zones).

Furthermore, we observe a characteristic feature of $\mathcal{P} \mathcal{T}$ symmetry: In general, more fundamental modes merge at smaller $\gamma$ than higher-order modes, because they are easier to break (see also [8]). Indeed, modes at the band edges (the more fundamental ones) are the first to merge [higher transmission zones at band edges in Figs. 7(a) and 7(b)]. Afterward, the passband centers (the less fundamental modes) begin to merge at greater $\gamma$ [Figs. 7(c) and 7(d)]. To illustrate, we show the fields of two band edge modes $\left(k_{0} \approx 0.58\right.$ and $0.42 \mathrm{rad} / \mu \mathrm{m}$ in Fig. 3 ) and two band center modes $\left(k_{0} \approx 0.52\right.$ and $\left.0.36 \mathrm{rad} / \mu \mathrm{m}\right)$. The band center modes present more modulation nodes than the edge modes, demonstrating a less fundamental pattern. Finally, at ever higher $\gamma$ the transmission picture will have fewer features and becomes largely blue: Almost all modes are broken, leading to negligible transmission.

Besides this general pattern, the behavior of the gap defect modes is more erratic: Some modes merge at small $\gamma$, while others remain in the band gap at high $\gamma$. We discuss these modes in detail in the next section.

\section{B. Local properties}

After this general $\mathcal{P} \mathcal{T}$ behavior of the TIFS structure for varying $\phi$, we focus on a few specific geometries (specific $\phi$ ) to distinguish more precise features. We show $T, R_{L}$, and $R_{R}$ as a function of $k_{0}$ and $\gamma$ in Fig. 8 for specific $\phi$ values. In these figures, left and right incidence corresponds to $\gamma>0$ and $\gamma<0$, respectively (the upper and lower halves of the graphs). Two band gaps are displayed, indicated by the two horizontal black arrows in Fig. 8(a). For certain values of $\phi$, an interface mode appears in these band gaps [denoted by the red arrows in Fig. 8(a) around $k_{0} \approx 0.425$ and $0.56 \mathrm{rad} / \mu \mathrm{m}$ at $\left.\gamma=0\right]$. 

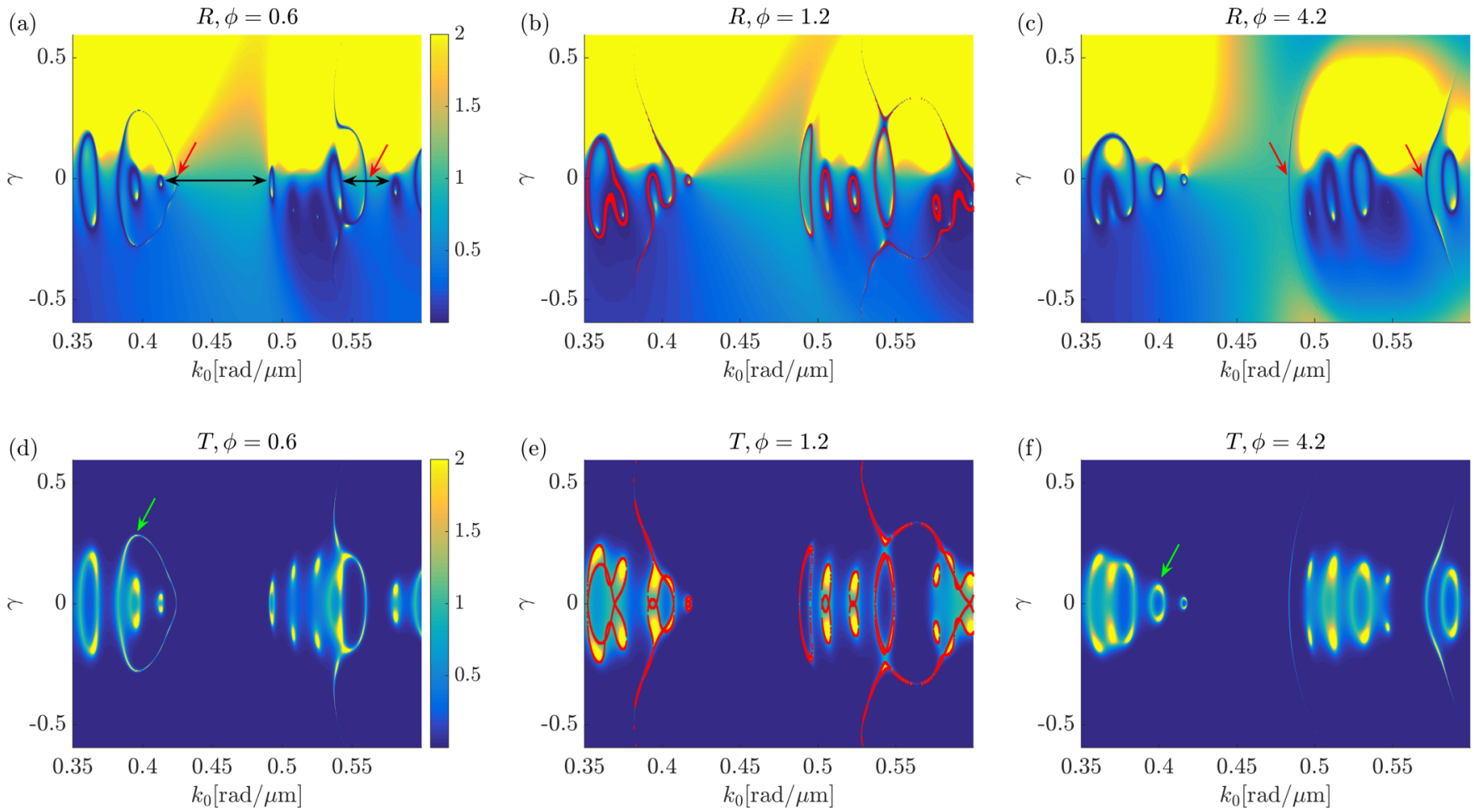

FIG. 8. (a)-(c) Reflection and (d)-(f) transmission for $P_{N, \phi}(N=50)$ as a function of $\gamma$ and $k_{0}$ and saturated to 2. (a) and (d) are with $\phi=0.6$, (b) and (e) with $\phi=1.2$, and (c) and (f) with $\phi=4.2$. Here $\gamma>0(\gamma<0)$ is for left (right) incidence. The two black double arrows in (a) indicate the band gaps. The two red arrows in (a) and (c) show the interfaces modes in these band gaps. The green arrow in (d) shows the merging of a band mode and an interface mode; in (f) it indicates two band modes merging. The red lines represent ATRs.

Most of the time $R_{L}>R_{R}$ [more yellow (light gray) in upper half] due to the presence of more gain on the left side $\left(\overleftarrow{X}_{N, \phi}\right)$ than on the right side (a consequence of the construction). We also note that, as usual in $\mathcal{P} \mathcal{T}$ symmetry, two modes tend to merge with one another at so-called exceptional points: Their eigenvalues become complex conjugates and they no longer lead to a transmission peak and only provide reflection afterward. Band modes merge with one another in each $\phi$ configuration [e.g., at the green arrow around $k_{0} \approx 0.4 \mathrm{rad} / \mu \mathrm{m}$ in Fig. 8(f)]. However, band-gap interface modes tend to merge with modes in the closest band [e.g., at the green arrow around $k_{0} \approx 0.4 \mathrm{rad} / \mu \mathrm{m}$ in Fig. 8(d)] or they merge with spectrally distant modes at large values of $\gamma$ [e.g., the interfaces modes that begin their trips at the red arrows around $k_{0} \approx 0.49$ and $0.57 \mathrm{rad} / \mu \mathrm{m}$ at $\gamma=0$ in Fig. 8(c)]. Note that we can expect that the system becomes unstable due to the large gain before the latter two modes merge.

During the merging process, the field profiles of the modes slowly become similar; see an example of an edge mode and a band mode combination in Fig. 9. The band mode (dotted red curve, middle), which is initially distributed along the structure, becomes more and more confined at the center (solid blue curve, bottom), similar to an edge mode profile. The edge mode evolves in the opposite way (dotted red and solid blue upper curves). In general, a band and edge mode merge at larger $\gamma$ than two band modes, because their profiles are quite different, and one needs more gain and loss to distort them. Conversely, two merging band modes are in general close (spectrally and with similar profiles), making them easier to match and merge at smaller $\gamma$.

Similar to other 1D $\mathcal{P} \mathcal{T}$ structures, we can observe anisotropic transmission resonances (ATRs) [43] or one-sided

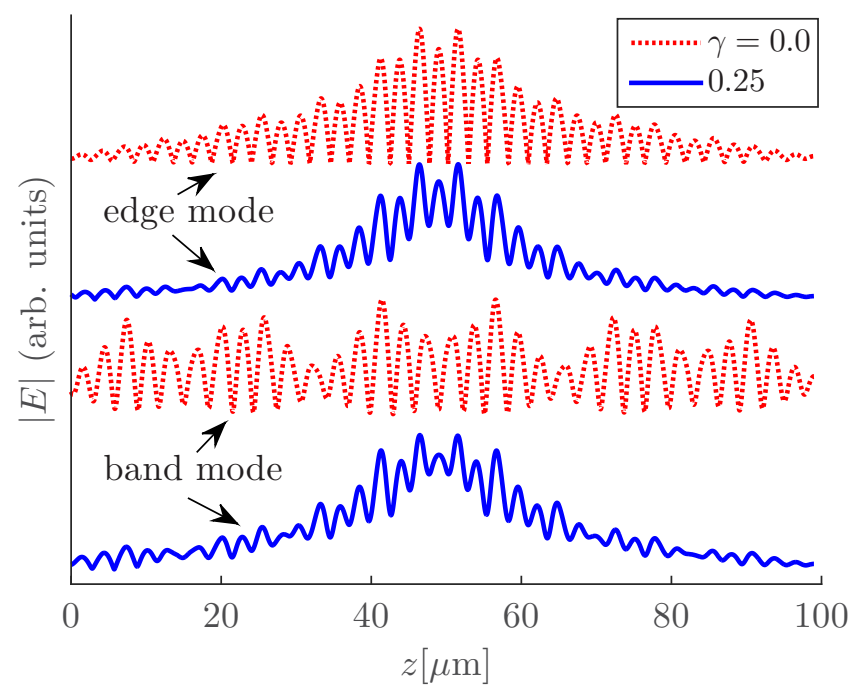

FIG. 9. Field profiles of an edge mode (two upper curves) and a band mode (two lower curves) that merge together. Dotted red curves show without gain and loss $\left[\gamma=0\right.$, around $k_{0} \approx 0.42$ and $0.38 \mathrm{rad} / \mu \mathrm{m}$, respectively, in Figs. 8(a) and 8(d)] and solid blue curves show just before their merging $\left(\gamma=0.25\right.$, around $k_{0} \approx 0.405$ and $0.39 \mathrm{rad} / \mu \mathrm{m}$, respectively, in Figs. 8(a) and 8(d)]. 

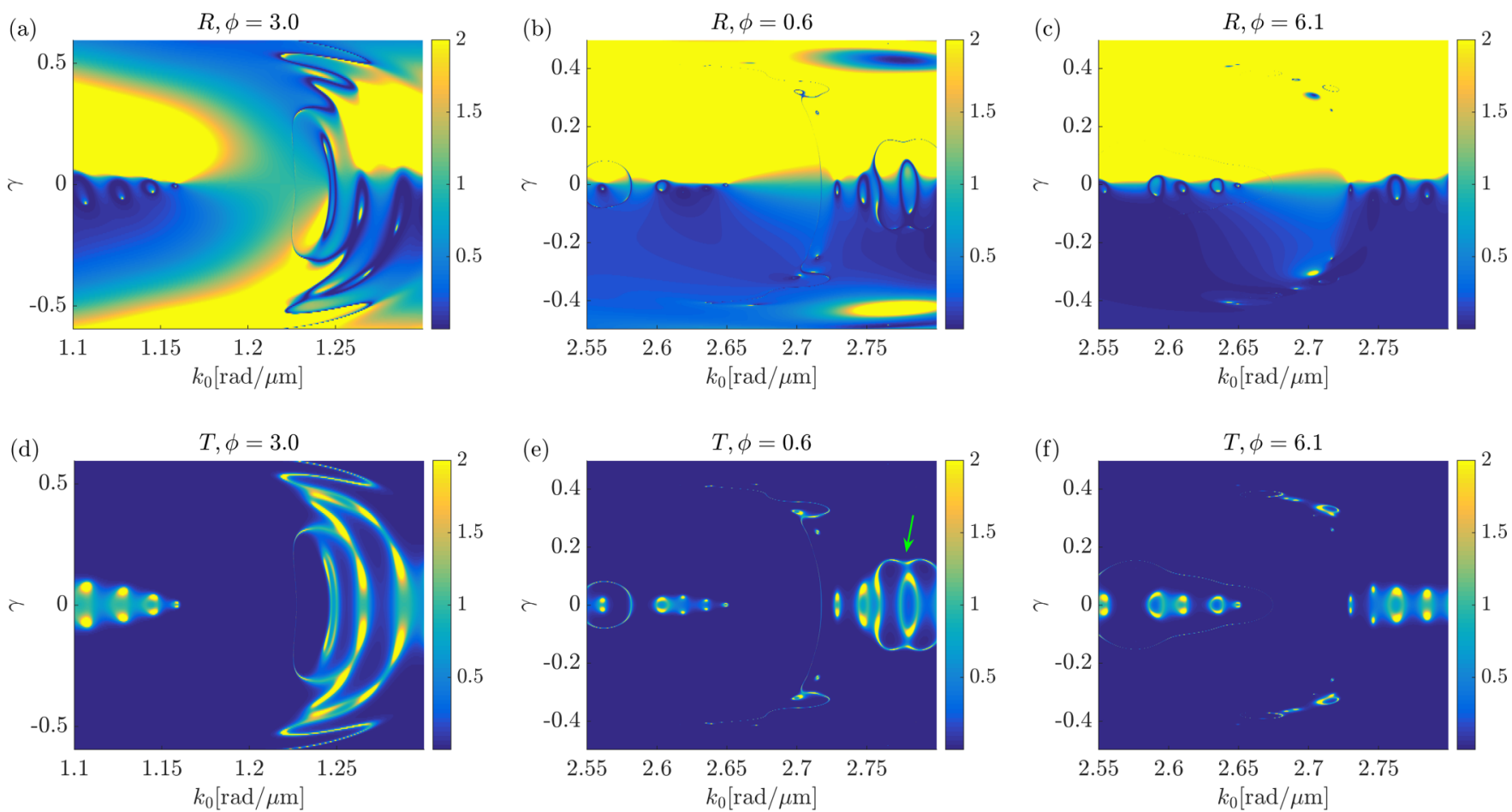

FIG. 10. (a)-(c) Reflection and (d)-(f) transmission for $P_{N, \phi}(N=50)$ as a function of $\gamma$ and $k_{0}$ and saturated to 2 for (a) and (d) $\phi=3.0$, (b) and (e) $\phi=0.6$, and (c) and (f) $\phi=6.1 \mathrm{rad}$. Here $\gamma>0(\gamma<0)$ is for left (right) incidence. The green arrow in (e) denotes a symmetry-recovery phenomenon.

invisibility, where $T=1$ [red lines in Fig. 8(e)] and one of the reflections is equal to zero [red lines in Fig. 8(b)]. These ATRs originate from the peaks of $T=1$ in the passive system where $R_{L}=R_{R}=0$. Upon the common introduction of gain and loss, the system distinguishes the left and right reflections, so only one is equal to zero and we detect the typical ATRs. In tight-binding approaches, e.g., with a limited number of cavities one can obtain analytical expressions for the ATRs [51]. Here, however, the analytics become unwieldy with many interfaces and we resort to numerical transfer matrix approaches, leading to fairly complex patterns of ATRs as a function of $\phi$ (Fig. 8).

In addition, we observe more exotic merging behaviors of the band modes [Figs. 10(a) and $10(\mathrm{~d})$ ] or interface modes [Figs. 10(b) and 10(e)]. Some islands of transmission appear in the band gap [around $\gamma \approx 0.32$ in Figs. 10(c) and 10(f)]. Furthermore, we find the so-called symmetry-recovery phenomenon [42] [denoted by the green arrow around $k_{0}=$ $2.77 \mathrm{rad} / \mu \mathrm{m}$ in Fig. 10(e)]: With tailored coupling between four modes, one can achieve the following sequence: two modes merge initially as $\gamma$ increases. However, when $\gamma$ continues to increase, they become real again (inverse exceptional point, around $\gamma=0.12$ ) and finally merge with their original $\mathcal{P} \mathcal{T}$ partners at greater $\gamma$. The fact that this appears is not strange, because there are many modes with many coupling constants, but still one needs a relatively delicate coupling balance, so it is uncommon to observe.

\section{Laser resonances}

Focusing on the merging behavior of an interface mode, we observe oscillations of the transmission, with peaks of very high intensity indicating lasing effects [yellow arrows in Figs. 11(a) and 11(b)]. These peaks are elegantly described with a Fabry-Pérot model of a cavity centered at the mirror plane. The phase-matching condition of a round-trip in the cavity (4) describes the presence and evolution of the modes. A quality factor of the cavity (5), as dictated by its inner reflections, gives us information about the strengths of the
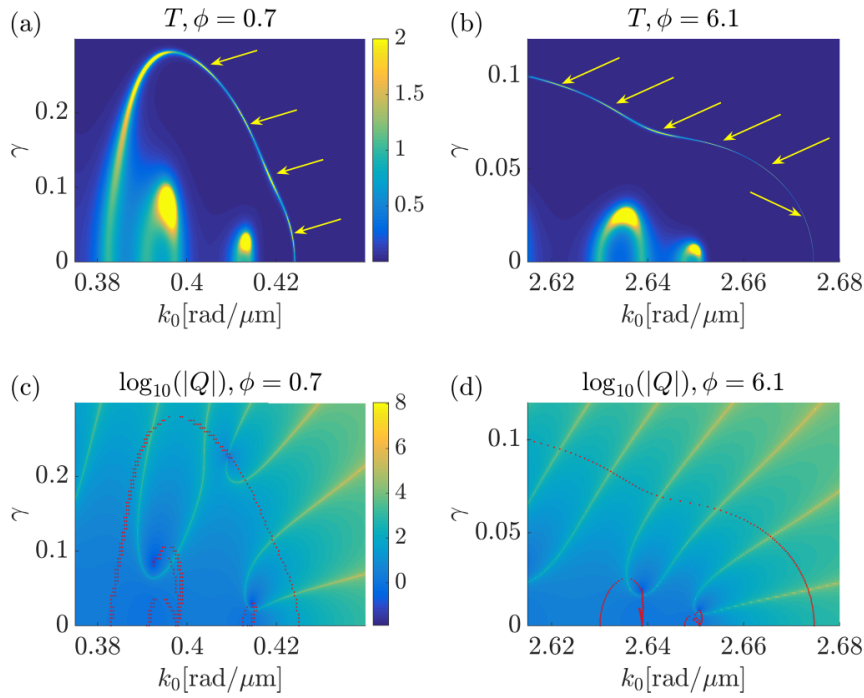

FIG. 11. (a) and (b) Transmission (saturated to 2) of $P_{N, \phi}$ $(N=50)$ as a function of $\gamma$ and $k_{0}$ via rigorous transfer matrix calculation. (c) and (d) Fabry-Pérot model with $\log _{10} Q$ [Eq. (5)] for the same structures. The red points represent constructive cavity interference, given by Eq. (4). 
resonances (at $\gamma \neq 0$ ) and so the intensity

$$
\begin{aligned}
& \theta_{\text {cav }}=2 m \pi, \quad m \in \mathbb{Z} \\
& Q=\frac{1}{1-\left|\overleftarrow{r}_{R}\right|\left|\vec{r}_{L}\right|}
\end{aligned}
$$

Equation (4) is indicated by the red dots in Figs. 11(c) and 11(d). The quality factor $Q$ depends on the amplitudes of the inner reflection coefficients of each part of $P_{N, \phi}$. We show $\log _{10}(Q)$ in Figs. 11(c) and 11(d). By comparing Figs. 11(a) and 11(b) and Figs. 11(c) and 11(d) we conclude that the modes are well described by this simple model: The alignment of phase matching (red dots) and high $Q$ [yellow (light gray) zones] in Figs. 11(c) and 11(d) indeed corresponds to the lasing resonances in Figs. 11(a) and 11(b).

By varying the crystal phase $\phi$ or by choosing different band gaps, we can observe various merging schemes of the interface mode. In Fig. 11(a), the variation of $k_{0}$ as a function of $\gamma$ of the interface mode branch is relatively small, leading to a succession of laser resonances closely spaced in frequency. In contrast, in Fig. 11(b), the variation of $k_{0}$ as a function of $\gamma$ along the branch is relatively large, leading to more spectrally distant resonances. The large number of possible merging scenarios allows us to spectrally spread or tighten the laser resonances and thus opens the way to laser resonance engineering. Furthermore, we can expect to take advantage of the field profile difference between band and interface modes in order to select which resonances will occur at smaller $\gamma$. Indeed, if we put gain and loss only in the layers close to the interface, we will reach the exceptional points of the interface modes at smaller $\gamma$ than for the band modes. Thus the interface mode should lase at smaller $\gamma$ and will not be perturbed by the band modes.

\section{CONCLUSION}

We studied a 1D photonic quasicrystal with topological features in a $\mathcal{P} \mathcal{T}$-symmetry context. We used the scattering characteristics to investigate various properties of this structure, especially the presence of interface modes in the band gaps. The structural degree of freedom $\phi$ (i.e., the crystal phase control variable) allows these modes to spectrally cross the band gap. These cycles are connected with the winding number of the band gap and the gap Chern number. Spectral jumps occur along these cycles for specific values of $\phi$, corresponding to the flipping of a letter in the crystal sequence. In addition, the symmetry of the gap modes are determined by these cycles and spectral jumps.

By adding gain and loss in a $\mathcal{P} \mathcal{T}$-symmetric fashion, we observed a set of complex behaviors that is not in simple correspondence with the passive case. Mostly, mode pairs merge at exceptional points under the action of $\mathcal{P} \mathcal{T}$ symmetry. This merging feature gives rise to high transmission peaks and even laser resonances. When $\gamma$ is beyond these exceptional points, the modes become complex and the transmission fades out. In a general pattern, we observe that the side modes of a band merge together at lower $\gamma$ than the less fundamental ones at the band center. We also observed a symmetry-recovery phenomenon, a complex merging scheme involving four band modes. The interface modes created in the band gaps merge with nearby band modes and present very high transmission at laserlike resonances. The spectral dispersion of these laser resonances can be controlled by the various involved parameters. In addition, we observed anisotropic transmission resonances where the transmission is unity and one of the reflections is equal to zero. The mix of order and disorder of the present system thus gives a useful basis for exploring how a variety of $\mathcal{P} \mathcal{T}$-symmetry-related effects are interrelated.

\section{ACKNOWLEDGMENT}

This work was supported by the Belgian Science Policy Office under the project "Photonics@be" (Grant No. P7-35) and by the Fonds National de Recherche Scientifique in Belgium.
[1] A. Guo, G. J. Salamo, D. Duchesne, R. Morandotti, M. VolatierRavat, V. Aimez, G. A. Siviloglou, and D. N. Christodoulides, Phys. Rev. Lett. 103, 093902 (2009).

[2] T. Kottos, Nat. Phys. 6, 166 (2010).

[3] L. Razzari and R. Morandotti, Nature (London) 488, 163 (2012).

[4] C. M. Bender and S. Boettcher, Phys. Rev. Lett. 80, 5243 (1998).

[5] C. M. Bender, Rep. Prog. Phys. 70, 947 (2007).

[6] A. Lupu, H. Benisty, and A. Degiron, Opt. Express 21, 21651 (2013).

[7] C. Huang, F. Ye, and X. Chen, Phys. Rev. A 90, 043833 (2014).

[8] H. Benisty, A. Lupu, and A. Degiron, Phys. Rev. A 91, 053825 (2015).

[9] R. El-Ganainy, K. G. Makris, D. N. Christodoulides, and Z. H. Musslimani, Opt. Lett. 32, 2632 (2007).

[10] N. X. A. Rivolta and B. Maes, J. Opt. Soc. Am. B 32, 1330 (2015).

[11] S. Phang, A. Vukovic, H. Susanto, T. M. Benson, and P. Sewell, J. Opt. Soc. Am. B 30, 2984 (2013).
[12] H. Benisty, A. Degiron, A. Lupu, A. D. Lustrac, S. Chénais, S. Forget, M. Besbes, G. Barbillon, A. Bruyant, S. Blaize, and G. Lérondel, Opt. Express 19, 18004 (2011).

[13] M. Kulishov and B. Kress, Opt. Express 20, 29319 (2012).

[14] L. Feng, Z. J. Wong, R.-M. Ma, Y. Wang, and X. Zhang, Science 346, 972 (2014).

[15] B. Peng, S. K. Özdemir, S. Rotter, H. Yilmaz, M. Liertzer, F. Monifi, C. M. Bender, F. Nori, and L. Yang, Science 346, 328 (2014).

[16] H. Ramezani, D. N. Christodoulides, V. Kovanis, I. Vitebskiy, and T. Kottos, Phys. Rev. Lett. 109, 033902 (2012).

[17] M. C. Zheng, D. N. Christodoulides, R. Fleischmann, and T. Kottos, Phys. Rev. A 82, 010103(R) (2010).

[18] K. G. Makris, R. El-Ganainy, D. N. Christodoulides, and Z. H. Musslimani, Phys. Rev. Lett. 100, 103904 (2008).

[19] A. V. Poshakinskiy, A. N. Poddubny, and A. Fainstein, Phys. Rev. Lett. 117, 224302 (2016).

[20] A. Jazaeri and I. I. Satija, Phys. Rev. E 63, 036222 (2001). 
[21] C. Hang, Y. V. Kartashov, G. Huang, and V. V. Konotop, Opt. Lett. 40, 2758 (2015).

[22] X.-F. Zhu, Opt. Express 23, 22274 (2015).

[23] S. Longhi, D. Gatti, and G. D. Valle, Phys. Rev. B 92, 094204 (2015).

[24] S. Longhi, D. Gatti, and G. D. Valle, Sci. Rep. 5, 13376 (2015).

[25] S. Phang, A. Vukovic, S. C. Creagh, P. D. Sewell, G. Gradoni, and T. M. Benson, Sci. Rep. 6, 20499 (2016).

[26] P. Ambichl, K. G. Makris, L. Ge, Y. D. Chong, A. D. Stone, and S. Rotter, Phys. Rev. X 3, 041030 (2013).

[27] M. Z. Hasan and C. L. Kane, Rev. Mod. Phys. 82, 3045 (2010).

[28] L. Lu, J. D. Joannopoulos, and M. Soljačić, Nat. Photon. 8, 821 (2014).

[29] D. Tanese, E. Gurevich, F. Baboux, T. Jacqmin, A. Lemaître, E. Galopin, I. Sagnes, A. Amo, J. Bloch, and E. Akkermans, Phys. Rev. Lett. 112, 146404 (2014).

[30] Y. E. Kraus, Y. Lahini, Z. Ringel, M. Verbin, and O. Zilberberg, Phys. Rev. Lett. 109, 106402 (2012).

[31] M. Verbin, O. Zilberberg, Y. E. Kraus, Y. Lahini, and Y. Silberberg, Phys. Rev. Lett. 110, 076403 (2013).

[32] M. Verbin, O. Zilberberg, Y. Lahini, Y. E. Kraus, and Y. Silberberg, Phys. Rev. B 91, 064201 (2015).

[33] A. Blanco-Redondo, I. Andonegui, M. J. Collins, G. Harari, Y. Lumer, M. C. Rechtsman, B. J. Eggleton, and M. Segev, Phys. Rev. Lett. 116, 163901 (2016).

[34] L.-J. Lang, X. Cai, and S. Chen, Phys. Rev. Lett. 108, 220401 (2012).

[35] H. Zhao, S. Longhi, and L. Feng, Sci. Rep. 5, 17022 (2015).
[36] K. Esaki, M. Sato, K. Hasebe, and M. Kohmoto, Phys. Rev. B 84, 205128 (2011).

[37] A. K. Harter, T. E. Lee, and Y. N. Joglekar, Phys. Rev. A 93, 062101 (2016).

[38] H. Schomerus, Opt. Lett. 38, 1912 (2013).

[39] T. E. Lee, Phys. Rev. Lett. 116, 133903 (2016).

[40] F. Baboux, E. Levy, A. Lemaître, C. Gómez, E. Galopin, L. Le Gratiet, I. Sagnes, A. Amo, J. Bloch, and E. Akkermans, Phys. Rev. B 95, 161114 (2017).

[41] E. Levy, A. Barak, A. Fisher, and E. Akkermans, arXiv: 1509.04028.

[42] N. X. A. Rivolta and B. Maes, Opt. Lett. 40, 3922 (2015).

[43] L. Ge, Y. D. Chong, and A. D. Stone, Phys. Rev. A 85, 023802 (2012).

[44] Optics of Aperiodic Structures: Fundamentals and Device Applications, edited by Luca Dal Negro (Pan Stanford, Singapore, 2013).

[45] X. Fu, Y. Liu, P. Zhou, and W. Sritrakool, Phys. Rev. B 55, 2882 (1997).

[46] http://camfr.sourceforge.net/

[47] J. Bellissard, B. Iochum, E. Scoppola, and D. Testard, Commun. Math. Phys. 125, 527 (1989).

[48] Y. Liu, X. Fu, W. Deng, and S. Wang, Phys. Rev. B 46, 9216 (1992).

[49] A. V. Poshakinskiy, A. N. Poddubny, and M. Hafezi, Phys. Rev. A 91, 043830 (2015).

[50] S. A. Skirlo, L. Lu, Y. Igarashi, Q. Yan, J. Joannopoulos, and M. Soljačić, Phys. Rev. Lett. 115, 253901 (2015).

[51] N. X. A. Rivolta and B. Maes, Phys. Rev. A 94, 053854 (2016). 